\title{
22-years magnetic cycle in polar activity of the Sun
}

\author{
V.I. Makarov ${ }^{1}$, A.G. Tlatov ${ }^{2}$, J. Singh and S.S. Gupta ${ }^{3}$ \\ ${ }^{1}$ Pulkovo Astronomical Observatory, St.Petersburg, Russia, email: makarov@gao.spb.ru \\ ${ }^{2}$ Kislovodsk Solar Station of the Pulkovo Observatory, Russia, email: solar@narzan.ru \\ ${ }^{3}$ Indian Institute of Astrophysics, Bangalore, India, email: jsingh@iiap.ernet.in
}

\begin{abstract}
We have digitized and processed the daily K-CaII-line full disk spectroheliograms from the archive of the Kodaikanal Observatory during 1907-1995. The programm has been worked out to determine the boundaries of the bright areas (plages, decayed plages, enhanced network features, K-CaII bright points and so on) with contrast that exceeded a level of the quiet Sun on the given value. About the $1.2 \times 10^{6} \mathrm{~K}$-CaII active regions of different scales were processed. The coordinates, areas, the tilt and latitude-time distributions of bright features were determined. At the high latitudes the K-CaII bright points form a polar branch of solar activity at the period between the polar magnetic field reversals. This polar activity shows both 11-year's and 22-year's cycles. We found that the polar K-CaII bright point cycles proceed on average 5.5 years the sunspot cycles.
\end{abstract}

I. During the last decade we work out a three-component model of the solar cycle, which includes the generation of the large-scale weak magnetic field as the basis for the sunspot and polar activity. From various series of synoptic data, we have received the strong relation between the sunspot cycle and the preceding large-scale weak magnetic field cycle with the 5-6 years delay (Makarov et al. (2001)). The K-CaII emission structures are the most significant manifestation of the global activity of the Sun.

The major goal of this paper is to present preliminary results from measurements of the $\mathrm{K}$-CaII emission areas on the daily ground-based spectroheliograms of the full solar disk. We attempted to separate the quiet network component from the quiet solar background as well as from plages by applying the new method developed by (Tlatov (2002)) for measuring solar feature areas. The K-CaII measurement program is to obtain the cycle variations of the "butterfly" diagrams at the low- and high latitudes, their asymmetry, K-CaII - indexes, tilt angle of the plages, active longitudes, biennial cycles during 19071995. Another goal of the research program is to compare our measurements with the traditional indicators of solar activity such as sunspot numbers, areas of plage regions, radio fluxes.

II. The observational data have been obtained by digitizing of K-CaII spectroheliograms taken at the Kodaikanal Observatory of the Indian Institute of Astrophysics (Bangalore). The spectroheliograph records a 6-cm image of the Sun at the K-CaII line on the photo plates. The 70-micron exit slit of the spectroheliograph separates $0.5 \AA$ band pass around $3933.67 \AA$, which includes $K_{232}$ (or K2r, K2v, and K3) profile. The image scale was about $0.032 \mathrm{~mm}$ arcsec $^{-1}$. The full set of the photo plates for 1907-1995 was digitized using the linear array with 900 pixels, which sample the K-CaII images at 2 arcsec interval.

III. All solar disk was divided into 15 segments to take account the effect of the limb darkening and non-uniform density of the plates. Each segment was divided into 20 parts on radius $\mathrm{r}$. The average intensity $I_{a v r}=I_{r}$ inside each of 300 segments was calculated. 


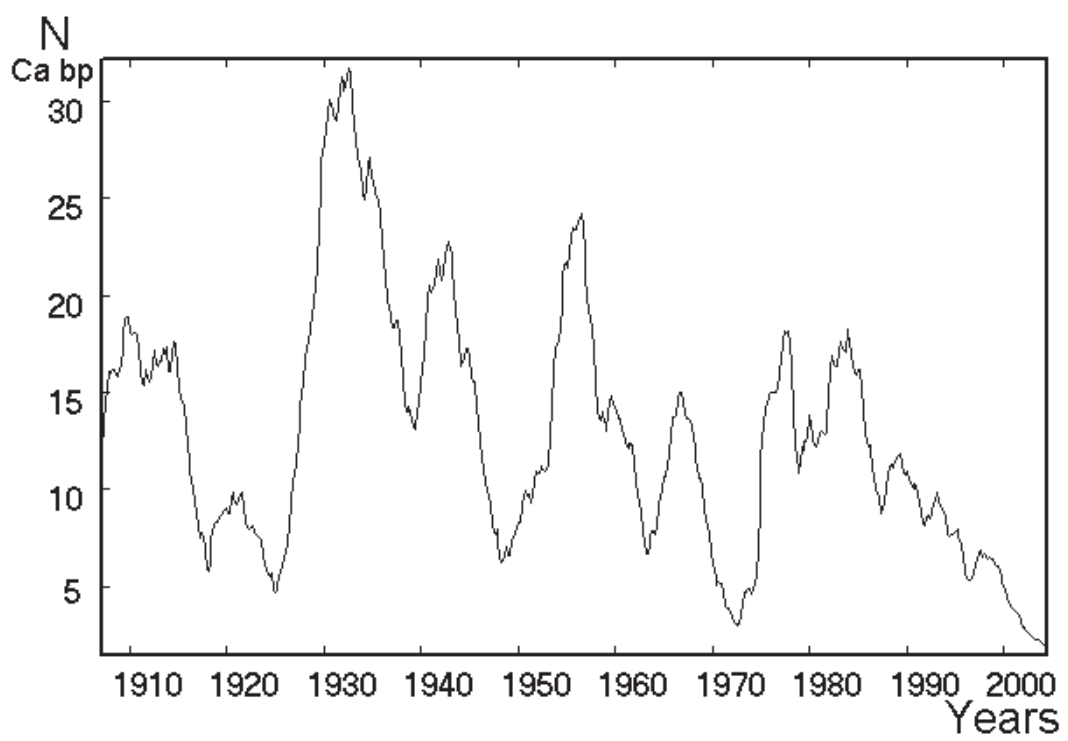

Figure 1. Monthly mean area of the K-CaII bright points at the latitudes above $50^{\circ}$ of the northern and southern hemispheres.

The average areas, coordinates, the size of the structures in the latitude and longitude directions, average and maximal contrast, perimeter, tilts of the $\mathrm{K}$-CaII bright regions were counted up. The bright areas with the size more than $20 \mathrm{mph}(\approx 12$ arcsec $)$ were processed. The areas of the $\mathrm{K}$-CaII bright regions were calculated as a fraction of the disc area, corrected for foreshortening, and expressed in $10^{-6}$ a part of the solar hemisphere.

IV. At the high latitudes the K-CaII bright points form a polar branch of solar activity at the period between the polar magnetic field reversals. This polar activity shows both 11-year's and 22-year's cycles. We found that the polar K-CaII bright point cycles proceed on average 5.5 years the sunspot cycles. Contrary to the sunspot activity, the intensity of the even cycles of polar activity exceeds the intensity of the even sunspot cycles. We found the two latitude drifts of the K-CaII activity during the polar cycle. The first type is related to the pole ward migration of the K-CaII activity in the beginning of polar cycle, and the second one connected with the equator ward migration from poles in the direction to the low latitudes after the polar magnetic field reversal.

\section{Acknowledgements}

This research was supported by the Russian Fund for Basic Research, grants 02-0216035 and 03-02-16091 and the National Program "Astronomy".

\section{References}

Makarov V.I., Tlatov A.G., Callebaut D.K., Obridko V.N. and B.D. Shelting 2001 Solar Phys. 198,409.

Tlatov A.G. 2002 in Proc. Pulkovo - Solar Conference - Solar Activity and Cosmic Rays After Polar Magnetic Field Reversal, 517. 